Amer H. H. Al-Khaladi

\title{
Meromorphic functions that share one value and the solution of Riccati differential equation
}

Received: 17 January 2012 / Accepted: 27 November 2012 / Published online: 18 December 2012

(C) The Author(s) 2012. This article is published with open access at Springerlink.com

Abstract In this paper, we shall give new examples on meromorphic functions that share one value with their first derivative and also give the solution for Riccati differential equation.

Mathematics Subject Classification 30D35

ريكاتي التفاضلية.

\section{Introduction and main results}

Throughout this note, the term "meromorphic" means meromorphic in the whole complex plane, and we shall use the standard notations of Nevanlinna theory of meromorphic functions [6]. For a meromorphic function $f$, let $T(r, f)$ denote the Nevanlinna characteristic of $f$ and let $S(r, f)$ be any quantity satisfying $S(r, f)=o(T(r, f))$ as $r \rightarrow \infty$, except possibly on a set of finite linear measure. A meromorphic function $a$ is said to be a small function of $f$ if $T(r, a)=S(r, f)$. We say that two non-constant meromorphic functions $f$ and $g$ share a value $a$ IM (ignoring multiplicities), if $f$ and $g$ have the same $a$-points. If $f$ and $g$ have the same $a$-points with the same multiplicities, we say that $f$ and $g$ share the value $a \mathrm{CM}$ (counting multiplicities). Let $n$ be a positive integer, we denote by $\left.N_{n}\right)\left(r, \frac{1}{f-a}\right)$ the counting function of $a$-points of $f$ with multiplicity $\leq n$ and by $N_{(n+1}\left(r, \frac{1}{f-a}\right)$ the counting function of $a$-points of $f$ with multiplicity $>n$. We denote by $\bar{N}_{=n}(r, f)$ or $\bar{N}_{\geq n}(r, f)$ the counting function of all the poles of $f$ which have the multiplicity $n$ or a multiplicity at least $n$, respectively. Each pole is counted only once in these counting functions (see [8]).

In [5], Gundersen proved the following theorem.

Theorem 1.1 If a non-constant meromorphic function $f$ and its derivative $f^{\prime}$ share two finite values $C M$, then $f=f^{\prime}$.

Theorem 1.1 was generalized for the higher-order derivatives:

Theorem 1.2 If a non-constant meromorphic function $f$ shares two distinct finite values $C M$ with its $k$-derivative $f^{(k)}$, then $f=f^{(k)}$.

A. H. H. Al-Khaladi ( $\otimes)$

Department of Mathematics, College of Basic Education, Tikrit University, P. O. Box 42, Tikrit, Iraq

E-mail: ameralkhaladi@yahoo.com 
This result is due to Frank and Ohlenroth [3] for the case that the shared values are nonzero and Frank and Weissenborn [4] for the general case. In addition, $\mathrm{Li}$ [7] gave an example which shows that condition $f$ and $f^{\prime \prime}$ have two shared CM is essential.

In [5], Gundersen gave the following example.

Example 1.3 [5] Let $f(z)=\frac{2 \ell}{1-b e^{-2 z}}$, where $\ell$ and $b$ are nonzero constants. It is easy to see that $f$ and $f^{\prime}$ share $0 \mathrm{CM}$ and $\ell \mathrm{IM}$, but $f \neq f^{\prime}$.

From Theorem 1.1 and Example 1.3, we can suggest the following question:

Question 1.4 If a non-constant meromorphic function $f$ and its derivative $f^{\prime}$ share the value $1 C M$, then $f-1=c\left(f^{\prime}-1\right)$, where $c$ is a nonzero constant?

We make an example which shows that the answer of this question is negative.

Example 1.5 Let

$$
f(z)=\frac{\int\left(1-c \mathrm{e}^{-\frac{1}{n} z}\right)^{n-1} \mathrm{~d} z}{\left(1-c \mathrm{e}^{-\frac{1}{n} z}\right)^{n}},
$$

if we let $w=1-c \mathrm{e}^{-\frac{1}{n} z}$, then

$$
\begin{aligned}
\int\left(1-c \mathrm{e}^{-\frac{1}{n} z}\right)^{n-1} \mathrm{~d} z & =n \int \frac{w^{n-1}}{1-w} \mathrm{~d} w \\
& =-n \int\left(w^{n-2}+w^{n-3}+\cdots+1+\frac{1}{w-1}\right) \mathrm{d} w \\
& =-n\left[\frac{w^{n-1}}{n-1}+\frac{w^{n-2}}{n-2}+\cdots+w+\ln (w-1)\right]+A \\
& =-n\left[\frac{\left(1-c \mathrm{e}^{-\frac{1}{n} z}\right)^{n-1}}{n-1}+\frac{\left(1-c \mathrm{e}^{-\frac{1}{n} z}\right)^{n-2}}{n-2}+\cdots+1-c \mathrm{e}^{-\frac{1}{n} z}\right]+z+A,
\end{aligned}
$$

where $A$ and $c \neq 0$ are constants. From (1.1), it is easy to see that $f$ and $f^{\prime}$ share $1 \mathrm{CM}$, but $f-1 \neq c\left(f^{\prime}-1\right)$, for any nonzero constant $c$. Indeed,

$$
f^{\prime}(z)-1=\frac{-c \mathrm{e}^{-\frac{1}{n} z}}{1-c \mathrm{e}^{-\frac{1}{n} z}}(f(z)-1) .
$$

Also, from (1.1), we see that

$$
f^{\prime}(z)-1=c \mathrm{e}^{-\frac{1}{n} z}\left(f^{\prime}(z)-f(z)\right) .
$$

This implies $f^{\prime}-1$ and $f^{\prime}-f$ share 0 CM. Further, it follows from (1.1) that $\bar{N}_{=n}(r, f) \neq S(r, f)$.

The purpose of this paper is to prove the following theorems:

Theorem 1.6 Let $f$ be a non-constant meromorphic function. If $f^{\prime}-1$ and $f^{\prime}-f$ share the value 0 CM and if $\bar{N}_{=n}(r, f) \neq S(r, f)$ for some positive integer $n$, then $f$ and $f^{\prime}$ share the value $1 C M$ and $f$ satisfies the identity (1.1).

Theorem 1.7 Let $f$ be a non-constant meromorphic function satisfying Riccati differential equation

$$
f^{\prime}=a_{0}+a_{1} f+a_{2} f^{2}
$$

where $a_{0}, a_{1}$ and $a_{2} \not \equiv 0$ are small functions of $f$. If $f^{\prime}-1$ and $f^{\prime}-f$ share the value 0 CM, then $f$ and $f^{\prime}$ share the value $1 C M$ and $f$ satisfies the identity (1.1) when $n=1$, i.e.,

$$
f(z)=\frac{z+A}{1-c \mathrm{e}^{-z}}
$$

where $A$ and $c \neq 0$ are constants. 
Theorem 1.8 Let $f$ be a non-constant meromorphic function. If $f^{\prime}-1$ and $f^{\prime}-f$ share the value 0 CM and if $f^{\prime}(z)-1=0$ when $f(z)-1=0$, then $f$ and $f^{\prime}$ share the value $1 C M$ and either $f$ satisfies the identity (1.1) or $f^{\prime}-f=e^{\beta}\left(f^{\prime}-1\right)$, where

$$
T\left(r, e^{\beta}\right) \leq \epsilon N(r, f)+S(r, f)
$$

for any positive real number $\epsilon$.

Theorem 1.9 Let $f$ be a non-constant meromorphic function satisfying Riccati differential equation

$$
f^{\prime}=a_{0}+a_{1} f+a_{2} f^{2}
$$

where $a_{0}, a_{1}$ and $a_{2} \not \equiv 0$ are small functions of $f$. If $f$ and $f^{\prime}$ share the value $1 I M$, then either

$$
f^{\prime}-1=a_{2}(f-1)^{2}
$$

or

$$
f^{\prime}-1=a_{2}(f-1)(f-z+A)
$$

where A is constant.

Remark 1.10 (1) If $a_{2} \equiv \frac{4}{c}$, then the general solution of (1.4) is

$$
f(z)=\frac{1+b+(b-1) c \mathrm{e}^{2 b \ell z}}{1-c \mathrm{e}^{2 b \ell z}}
$$

where $b, c$ and $\ell$ are nonzero constants and $b^{2} \ell=-1$.

(2) The formula (1.5) may be put in the form

$$
-\frac{f^{\prime}-1}{(f-z+A)^{2}}=-a_{2}-\frac{a_{2}(z-A-1)}{(f-z+A)},
$$

or

$$
y^{\prime}+a_{2}(z-A-1) y=-a_{2},
$$

where $y=\frac{1}{f-z+A}$. The general solution of (1.6) is

$$
I y=-\int a_{2} I d z+\lambda
$$

where $I=\mathrm{e}^{\int a_{2}(z-A-1) \mathrm{d} z}$ and $\lambda$ is a constant.

(3) Special case of (2), if $a_{2} \equiv \frac{-1}{z-A}$, then the general solution of (1.5) is

$$
f(z)=\frac{z-A}{1-c \mathrm{e}^{-z}}
$$

where $A$ and $c \neq 0$ are constants. 


\section{Lemmas}

For the proof of our theorems, we need the following lemmas.

Lemma 2.1 [9] Let $f$ be a meromorphic function such that $f^{(k)}$ is not constant. Then

$$
T(r, f) \leq N\left(r, \frac{1}{f-1}\right)+\bar{N}\left(r, \frac{1}{f^{(k)}-1}\right)+\bar{N}(r, f)-N_{0}\left(r, \frac{1}{f^{(k+1)}}\right)+S(r, f),
$$

where $N_{0}\left(r, \frac{1}{f^{(k+1)}}\right)$ denotes the counting function of the zeros of $f^{(k+1)}$ that are not zeros of $f^{(k)}$, where these zeros are counted according to their multiplicity.

Lemma 2.2 [2] Let $k$ be a positive integer, and let $f$ be a meromorphic function such that $f^{(k)}$ is not constant. Then either

$$
\left(f^{(k+1)}\right)^{k+1}=c\left(f^{(k)}-\lambda\right)^{k+2},
$$

for some nonzero constant c, or

$$
k N_{1)}(r, f) \leq \bar{N}_{(2}(r, f)+N_{1)}\left(r, \frac{1}{f^{(k)}-\lambda}\right)+\bar{N}\left(r, \frac{1}{f^{(k+1)}}\right)+S(r, f),
$$

where $\lambda$ is a constant.

The following lemma essentially belongs to [1]. For completeness, we give its proof here.

Lemma 2.3 [1] Let $k$ be a positive integer, and let $f$ be a non-constant meromorphic function. Then either (2.2) holds, or

$$
f(z)=\frac{-(k+1)^{k+1}}{c k ![z+(k+1) A]}+\lambda \frac{z^{k}}{k !}+p_{k-1}(z)
$$

where $c \neq 0, A, \lambda$ are constants and $p_{k-1}$ is a polynomial of degree at most $k-1$.

Proof If $f^{(k)}$ is a constant, then $f$ is a polynomial of degree at most $k$ and so $N_{1)}(r, f)=S(r, f)$. In this case (2.2) holds. Next we suppose that $f^{(k)}$ is not constant. By Lemma 2.2, if (2.2) is not true then we have (2.1). Writing (2.1) as

$$
\left(\frac{f^{(k+1)}}{f^{(k)}-\lambda}\right)^{k+1}=c\left(f^{(k)}-\lambda\right) .
$$

Differentiating (2.4), we obtain

$$
(k+1)\left(\frac{f^{(k+1)}}{f^{(k)}-\lambda}\right)^{k}\left(\frac{f^{(k+1)}}{f^{(k)}-\lambda}\right)^{\prime}=c f^{(k+1)} .
$$

Combining this with (2.4) yields

$$
\left(\frac{f^{(k+1)}}{f^{(k)}-\lambda}\right)^{-2}\left(\frac{f^{(k+1)}}{f^{(k)}-\lambda}\right)^{\prime}=\frac{1}{k+1} .
$$

Integrating this once and then using (2.4), we have

$$
f^{(k)}(z)-\lambda=\frac{1}{c}\left[\frac{-(k+1)}{z+A(k+1)}\right]^{k+1} .
$$

By integrating (2.5) $k$ times we arrive at (2.3). 


\section{The Proof of Theorems}

\subsection{Proof of Theorem 1.6}

Since $f^{\prime}-1$ and $f^{\prime}-f$ share $0 \mathrm{CM}$, there is an entire function $\beta$ such that

$$
\frac{f^{\prime}-f}{f^{\prime}-1}=\mathrm{e}^{\beta}
$$

Suppose that $z_{\infty}$ is a pole of $f$ with the multiplicity $n \geq 1$. Then the Laurent expansion of $f$ about $z_{\infty}$ is

$$
f(z)=\frac{a_{n}}{\left(z-z_{\infty}\right)^{n}}+\frac{a_{n-1}}{\left(z-z_{\infty}\right)^{n-1}}+\cdots, \quad a_{n} \neq 0 .
$$

Hence

$$
f^{\prime}(z)=\frac{-n a_{n}}{\left(z-z_{\infty}\right)^{n+1}}+\frac{(1-n) a_{n-1}}{\left(z-z_{\infty}\right)^{n}}+\cdots
$$

From (3.2) and (3.3), we find that

$$
f^{\prime}(z)-f(z)=\frac{-n a_{n}}{\left(z-z_{\infty}\right)^{n+1}}+\frac{(1-n) a_{n-1}-a_{n}}{\left(z-z_{\infty}\right)^{n}}+\cdots
$$

It follows from (3.1), (3.3) and (3.4) that

$$
\mathrm{e}^{\beta(z)}=1+\frac{1}{n}\left(z-z_{\infty}\right)+\cdots
$$

Differentiating (3.5) we obtain

$$
\beta^{\prime}(z) \mathrm{e}^{\beta(z)}=\frac{1}{n}+\cdots
$$

and eliminating $\mathrm{e}^{\beta}$ between (3.5) and (3.6) gives

$$
\beta^{\prime}(z)=\frac{1}{n}+\cdots
$$

We distinguish the following two cases.

Case 1. $\beta^{\prime} \equiv \frac{1}{n}$ for some positive integer $n$. Then from this and (3.1) we have

$$
\frac{f^{\prime}-f}{f^{\prime}-1}=\frac{1}{c} \mathrm{e}^{\frac{1}{n} z}
$$

where $c$ is a nonzero constant. Writing (3.8) as

$$
f^{\prime}+\frac{c \mathrm{e}^{-\frac{1}{n} z}}{1-c \mathrm{e}^{-\frac{1}{n} z}} f=\frac{1}{1-c \mathrm{e}^{-\frac{1}{n} z}} .
$$

From this, it is easy to see that

$$
\frac{d}{\mathrm{~d} z}\left[\left(1-c \mathrm{e}^{-\frac{1}{n} z}\right)^{n} f\right]=\left(1-c \mathrm{e}^{-\frac{1}{n} z}\right)^{n-1} .
$$

By integration, we get

$$
f(z)=\frac{\int\left(1-c \mathrm{e}^{-\frac{1}{n} z}\right)^{n-1} \mathrm{~d} z}{\left(1-c \mathrm{e}^{-\frac{1}{n} z}\right)^{n}},
$$

This is (1.1). From this, it is easy to see that $f$ and $f^{\prime}$ share $1 \mathrm{CM}$. 
Case 2. $\beta^{\prime} \not \equiv \frac{1}{n}$ for all positive integer $n$. Then

$$
\bar{N}_{=n}(r, f) \leq N\left(r, \frac{1}{\beta^{\prime}-\frac{1}{n}}\right) \leq T\left(r, \beta^{\prime}\right)+O(1)=S\left(r, \mathrm{e}^{\beta}\right) .
$$

From (3.1), we find that

$$
\begin{aligned}
T\left(r, \mathrm{e}^{\beta}\right) & \leq T\left(r, f^{\prime}-f\right)+T\left(r, f^{\prime}-1\right)+O(1) \\
& \leq T(r, f)+2 T\left(r, f^{\prime}\right)+O(1) \\
& \leq 5 T(r, f)+S(r, f) .
\end{aligned}
$$

Therefore, this and (3.10) give that $\bar{N}_{=n}(r, f)=S(r, f)$ for all positive integer $n$ which contradicts our assumption.

\subsection{Proof of Theorem 1.7}

From Riccati differential equation, it is easy to conclude that $N_{(2}(r, f)+m(r, f)=S(r, f)$. This implies $T(r, f)=N_{1)}(r, f)+S(r, f)$. Hence $N_{1)}(r, f) \neq S(r, f)$, i.e., $N_{=1}(r, f) \neq S(r, f)$. By Theorem 1.6, we have (1.1) when $n=1$, i.e., (1.2) holds. Substituting (1.2) into $f^{\prime}=a_{0}+a_{1} f+a_{2} f^{2}$, we can deduce that

$$
c^{2} a_{0} \mathrm{e}^{-2 z}+c\left[z+A+1-2 a_{0}-a_{1}(z+A)\right] \mathrm{e}^{-z}+a_{2}(z+A)^{2}+a_{1}(z+A)+a_{0}-1 \equiv 0 .
$$

If $a_{0} \not \equiv 0$, then from the last equation we obtain $T\left(r, \mathrm{e}^{-z}\right)=S\left(r, \mathrm{e}^{-z}\right)$ which is impossible. Therefore, we have $a_{0} \equiv 0$. Similarly, we can conclude that

$z+A+1-a_{1}(z+A) \equiv 0$ and $a_{2}(z+A)^{2}+a_{1}(z+A)-1 \equiv 0$,

which implies $a_{1}(z)=\frac{z+A+1}{z+A}$ and $a_{2}(z)=\frac{-1}{z+A}$.

\subsection{Proof of Theorem 1.8}

If $f$ does not satisfy the identity (1.1), then by Theorem 1.6 we find that $\bar{N}_{=n}(r, f)=S(r, f)$ for all positive integer $n$. Thus

$$
\begin{aligned}
\bar{N}(r, f) & =\sum_{n=1}^{m-1} \bar{N}_{=n}(r, f)+\bar{N}_{\geq m}(r, f) \leq S(r, f)+\frac{1}{m} N(r, f) \\
& \leq \in N(r, f)+S(r, f),
\end{aligned}
$$

for any positive real number $\epsilon$. We rewrite (3.1) in the form

$$
\frac{f-1}{f^{\prime}-1}=1-\mathrm{e}^{\beta}
$$

If $z_{0}$ is a zero of $f^{\prime}-1$ with multiplicity $p$, then the Taylor expansion of $f^{\prime}-1$ about $z_{0}$ is

$$
f^{\prime}(z)-1=a_{p}\left(z-z_{0}\right)^{p}+\cdots, \quad a_{p} \neq 0 .
$$

Since $f^{\prime}-1$ and $f^{\prime}-f$ share $0 \mathrm{CM}$,

$$
f^{\prime}(z)-f(z)=b_{p}\left(z-z_{0}\right)^{p}+\cdots, \quad b_{p} \neq 0,
$$

and eliminating $f^{\prime}(z)$ between this and (3.13) we obtain

$$
f(z)-1=\left(a_{p}-b_{p}\right)\left(z-z_{0}\right)^{p}+\cdots
$$

Differentiating (3.14) we get

$$
f^{\prime}(z)=p\left(a_{p}-b_{p}\right)\left(z-z_{0}\right)^{p-1}+\cdots
$$


Together with (3.13) we have $p=1$. It follows from this, (3.13) and (3.14) that $z_{0}$ is a simple zero of $f^{\prime}-1$ and $f-1$. If $z_{1}$ is a zero of $f-1$ with multiplicity $q$, then

$$
f(z)-1=c_{q}\left(z-z_{0}\right)^{q}+\cdots
$$

Differentiating this we get

$$
f^{\prime}(z)=q c_{q}\left(z-z_{0}\right)^{q-1}+\cdots
$$

Since $f^{\prime}(z)-1=0$ when $f(z)-1=0$, we see that $q=1$. Therefore, we find that $f^{\prime}-1$ and $f-1$ share 0 CM. From this and (3.12), it is easy to conclude that

$$
\bar{N}(r, f)=\bar{N}\left(r, \frac{1}{\mathrm{e}^{\beta}-1}\right) .
$$

From this and the second fundamental theorem for $\mathrm{e}^{\beta}$, we have

$$
T\left(r, \mathrm{e}^{\beta}\right)=\bar{N}(r, f)+S(r, f) .
$$

Together with (3.11) we find that (1.3) holds.

\subsection{Proof of Theorem 1.9}

Suppose $z_{2}$ be a zero of $f^{\prime}-1$ and $a_{j}\left(z_{2}\right) \neq 0, \infty(j=0,1,2)$. Since $f$ and $f^{\prime}$ share the value $1 \mathrm{IM}$, we know that $z_{2}$ is a simple zero of $f-1$. From this and Riccati differential equation, we deduce that

$$
\left(a_{0}+a_{1}+a_{2}\right)\left(z_{2}\right)=1 .
$$

If $a_{0}+a_{1}+a_{2} \not \equiv 1$, then

$$
\begin{aligned}
\bar{N}\left(r, \frac{1}{f^{\prime}-1}\right)=N\left(r, \frac{1}{f-1}\right) & \leq N\left(r, \frac{1}{a_{0}+a_{1}+a_{2}-1}\right)+S(r, f) \\
& \leq T\left(r, a_{0}+a_{1}+a_{2}\right)+S(r, f) \\
& \leq T\left(r, a_{0}\right)+T\left(r, a_{1}\right)+T\left(r, a_{2}\right)+S(r, f) \\
& =S(r, f) .
\end{aligned}
$$

From Riccati differential equation, it is easy to conclude that

$$
N_{(2}(r, f)+m(r, f)=S(r, f) .
$$

Combining (3.16), (3.17) and Lemma 2.1, we obtain

$$
\begin{aligned}
T(r, f) & \leq N\left(r, \frac{1}{f-1}\right)+\bar{N}\left(r, \frac{1}{f^{\prime}-1}\right)+\bar{N}(r, f)-N_{0}\left(r, \frac{1}{f^{\prime \prime}}\right)+S(r, f) \\
& =N_{1)}(r, f)-N_{0}\left(r, \frac{1}{f^{\prime \prime}}\right)+S(r, f) .
\end{aligned}
$$

Consequently,

$$
N_{0}\left(r, \frac{1}{f^{\prime \prime}}\right)=S(r, f)
$$

Applying Lemma 2.3 to $k=1$ and $\lambda=1$ we get either

$$
N_{1)}(r, f) \leq \bar{N}_{(2}(r, f)+N_{1)}\left(r, \frac{1}{f^{\prime}-1}\right)+\bar{N}\left(r, \frac{1}{f^{\prime \prime}}\right)+S(r, f),
$$

or

$$
f(z)=\frac{-4}{c(z+2 A)}+z+B
$$


where $A, B$ and $c \neq 0$ are constants. If we combine (3.19), (3.17) and (3.16), we see that

$$
T(r, f) \leq \bar{N}\left(r, \frac{1}{f^{\prime \prime}}\right)+S(r, f)
$$

Together with (3.18), we find that

$$
\begin{aligned}
T(r, f) & \leq \bar{N}_{(2}\left(r, \frac{1}{f^{\prime}-1}\right)+\bar{N}_{0}\left(r, \frac{1}{f^{\prime \prime}}\right)+S(r, f) \\
& =\bar{N}_{(2}\left(r, \frac{1}{f^{\prime}-1}\right)+S(r, f) .
\end{aligned}
$$

This and (3.16) yield that $T(r, f)=S(r, f)$ a contradiction. Thus (3.19) does not hold. By (3.20) we find that

$$
f(z)-1=\frac{-4-c(z+2 A)(z+B)}{c(z+2 A)}
$$

and

$$
f^{\prime}(z)-1=\frac{-4}{c(z+2 A)^{2}} .
$$

So $f$ and $f^{\prime}$ can not share $1 \mathrm{IM}$ which contradicts the condition of Theorem 1.9. Therefore, we have $a_{0}+a_{1}+$ $a_{2} \equiv 1$. Substituting this into Riccati differential equation gives

$$
f^{\prime}-1=a_{2}(f-1)\left(f+1+\frac{a_{1}}{a_{2}}\right) .
$$

If $1+\frac{a_{1}}{a_{2}}=-1$ or $1+\left(\frac{a_{1}}{a_{2}}\right)^{\prime}=0$, then we obtain the conclusion (1.4) or (1.5) respectively. Otherwise, we conclude that $N\left(r, \frac{1}{f+1+\frac{a_{1}}{a_{2}}}\right)=S(r, f)$. Indeed, if $f+1+\frac{a_{1}}{a_{2}}$ has a zero of multiplicity $p$ at $z_{0}$, say, then $\frac{1}{a_{2}} \frac{f^{\prime}-1}{f-1}$ has a zero of multiplicity $p$ at $z_{0}$ as well. Then we must have either $a_{2}\left(z_{0}\right)=\infty$, or $f^{\prime}\left(z_{0}\right)=f\left(z_{0}\right)=1$, or $f\left(z_{0}\right)=\infty$. If $f\left(z_{0}\right)=\infty$, then $a_{2}$ has a pole of multiplicity $p+1$ at $z_{0}$, while if $f^{\prime}\left(z_{0}\right)=f\left(z_{0}\right)=1$, then $2+\frac{a_{1}}{a_{2}}$ has a zero of multiplicity 1 at $z_{0}$ and $1+\left(\frac{a_{1}}{a_{2}}\right)^{\prime}$ has a zero of multiplicity $\min \{p-1, p+1-s\}$ at $z_{0}$, where $s$ denotes the possible multiplicity of the pole of $a_{2}$ at $z_{0}$. In the remaining case $a_{2}$ must have a pole of multiplicity $p$ at $z_{0}$. Therefore

$$
\begin{aligned}
N\left(r, \frac{1}{f+1+\frac{a_{1}}{a_{2}}}\right) & \leq N\left(r, a_{2}\right)+N\left(r, \frac{1}{2+\frac{a_{1}}{a_{2}}}\right)+N\left(r, \frac{1}{1+\left(\frac{a_{1}}{a_{2}}\right)^{\prime}}\right) \\
& =S(r, f) .
\end{aligned}
$$

Writing (3.21) as

$$
\frac{\left(f+1+\frac{a_{1}}{a_{2}}\right)^{\prime}}{f+1+\frac{a_{1}}{a_{2}}}-\frac{1+\left(\frac{a_{1}}{a_{2}}\right)^{\prime}}{f+1+\frac{a_{1}}{a_{2}}}=a_{2}(f-1) .
$$

It follows from this and (3.17) that, if $1+\left(\frac{a_{1}}{a_{2}}\right)^{\prime} \not \equiv 0$, then

$$
m\left(r, \frac{1}{f+1+\frac{a_{1}}{a_{2}}}\right)=S(r, f) .
$$

Together with (3.22) we deduce that $T(r, f)=S(r, f)$, a contradiction. Hence we obtain that $1+\left(\frac{a_{1}}{a_{2}}\right)^{\prime} \equiv 0$. By integration, we get $\frac{a_{1}}{a_{2}}=-z+A$. From this and (3.21), we arrive at the conclusion (1.5).

Acknowledgments I am grateful to the referees for valuable suggestions and comments.

Open Access This article is distributed under the terms of the Creative Commons Attribution License which permits any use, distribution, and reproduction in any medium, provided the original author(s) and the source are credited. 


\section{References}

1. Al-Khaladi, A.H.H.: Meromorphic functions that share one small function with their $k$-th derivative. Analysis 31, 341-354 (2011)

2. Al-Khaladi, A.H.H.: On meromorphic functions that share one value with their derivative. Analysis 25, 131-140 (2005)

3. Frank, G.; Ohlenroth, W.: Meromorphe Funktionen, die mit einer ihrer Ableitungen Werte teilen. Complex Variables 6, 23-37 (1986)

4. Frank, G.; Weissenborn, G.: Meromorphe Funktionen, die mit einer ihrer Ableitungen Werte teilen. Complex Variables 7, 33-43 (1986)

5. Gundersen, G.G.: Meromorphic functions that share two finite values with their derivative. Pac. J. Math. 105, 299-309 (1983)

6. Hayman, W.K.: Meromorphic Functions. Clarendon Press, Oxford (1964)

7. Li, P.; Yang, C.C.: Value sharing of an entire function and its derivatives. J. Math. Soc. Japan 51, 781-799 (1999)

8. Mues, E.; Reinders, M.:On a Questuion of C. C. Yang. Complex Variables 34, 171-179 (1997)

9. Yang, C.C.; Yi, H.X.: Uniqueness theory of meromorphic functions, Kluwer, Dordrecht (2004) 